\title{
Binary Populations of Carbon-Enhanced Metal-Poor Stars
}

\author{
Robert G. Izzard ${ }^{\mathrm{A}, \mathrm{B}, \mathrm{F}}$, Evert Glebbeek ${ }^{\mathrm{B}, \mathrm{C}}$, Richard J. Stancliffe $e^{\mathrm{D}, \mathrm{E}}$, \\ and Onno Pols ${ }^{\mathrm{B}}$ \\ A Institut d'Astronomie et d'Astrophysique, Université Libre de Bruxelles, \\ Boulevard du Triomphe, B-1050 Brussels, Belgium \\ B Sterrenkundig Instituut, Universiteit Utrecht, P.O. Box 80 000, NL-3508 TA Utrecht, The Netherlands \\ ${ }^{\mathrm{C}}$ Department of Physics and Astronomy, McMaster University, Hamilton, Ontario, L8S 4M1, Canada \\ D School of Mathematical Sciences, PO Box 28M, Monash University, Victoria 3800, Australia \\ ${ }^{\mathrm{E}}$ Institute of Astronomy, University of Cambridge, Madingley Road, Cambridge, CB3 OHA, UK. \\ F Corresponding author. Email: robert.izzard@ulb.ac.be
}

Received 2008 December 14, accepted 2009 March 25

\begin{abstract}
We construct binary-star population nucleosynthesis models of carbon-enhanced metal-poor (CEMP) stars. We compare the CEMP to extremely metal-poor (EMP) ratio of our models to the observed ratio and find it is an order of magnitude too small. Through an increase in the efficiency of third dredge-up in low-mass, low-metallicity, thermally-pulsing Asymptotic Giant Branch (TP-AGB) stars our models better match the observations.
\end{abstract}

Keywords: Galaxy: halo — nucleosynthesis — stars: abundances — stars: AGB — stars: binaries

\section{Introduction}

The carbon-enhanced metal-poor stars (CEMPs) are Galactic halo objects which are observed to be iron poor $([\mathrm{Fe} / \mathrm{H}] \lesssim-2)$, relatively carbon rich $([\mathrm{C} / \mathrm{Fe}] \geq 1)$ and mostly giant and turn-off stars $(\log g \lesssim 4)$. Their mass is around $0.85 \mathrm{M}_{\odot}$, as dictated by the age of the halo. Canonical models of $\sim 0.85 \mathrm{M}_{\odot}$ single stars do not predict such a large carbon enhancement at any phase of their evolution. Instead, it seems likely that most CEMPs form in binary systems as a result of mass transfer from a carbon-rich TP-AGB star which is now an unseen white dwarf. Radial velocity surveys concur, at least for those CEMPs which are also rich in $s$-process elements (Tsangarides, Ryan \& Beers 2004; Lucatello et al. 2005b). According to detailed TP-AGB models the primary star should have had a mass in the range 1.2 to $3 \mathrm{M}_{\odot}$, in order to undergo efficient third dredge-up of carbon without hot-bottom burning (which converts carbon to nitrogen, e.g. Karakas \& Lattanzio 2007). For a review of the properties of carbon stars see, e.g., Wallerstein \& Knapp (1998) or Abia et al. (2003).

The $\mathrm{CH}$ stars are thought to be the higher metallicity $([\mathrm{Fe} / \mathrm{H}] \sim-1)$ equivalents of the CEMPs because they form by a similar binary-accretion mechanism. They make up about $1 \%$ of the giant population (Luck \& Bond 1991), in rough agreement with our theoretical estimates based on binary population synthesis (see Section 2). By contrast 9-30\% of the EMP giant population are CEMPs (Frebel et al. 2006; Lucatello et al. 2006; Suda et al. 2008), in disagreement with our standard binary population models.

Recent studies suggest that an initial-mass function (IMF) quite different to that of the solar neighbourhood is responsible for the large CEMP/EMP number ratio (e.g. Lucatello et al. 2005a; Komiya et al. 2007). We attempt to explain the CEMP/EMP number ratio without a change in the initial distributions of stellar masses and initial periods from those found in the solar neighbourhood.

Many of the physical parameters in our binary-star model are quite uncertain, but we show that most are not important in calculation of the CEMP/EMP number ratio. The parameters which matter most are those that affect the efficiency of third dredge-up in low-mass stars.

\section{Binary Population Models}

We base our population synthesis models on the rapid binary-star evolution and nucleosynthesis models of Hurley, Tout \& Pols (2002), Izzard et al. (2004) and Izzard et al. (2006). Our nucleosynthesis algorithm has been updated to better model first dredge-up in low-metallicity stars which have accreted carbon-rich material from a companion. Third dredge-up is modelled as in Karakas, Lattanzio \& Pols (2002) using the following parameters: $M_{\mathrm{c}, \min }$, the minimum core mass for third dredge-up; and $\lambda$, the ratio of the mass of material which is dredged up at a given pulse to the mass by which the core grew during the preceding interpulse period.

We also include the correction factors $\Delta M_{\mathrm{c}, \min }$ and $\lambda_{\min }$ which were introduced in Izzard et al. (2004) in order to match our synthetic TP-AGB models to observed carbon-star luminosity functions in the Magellanic Clouds. These alter the prescription of Karakas et al. (2002) such that $M_{\mathrm{c}, \min }=M_{\mathrm{c}, \min }^{\text {Karakas }}+\Delta M_{\mathrm{c}, \text { min }}$ and $\lambda=\max \left(\lambda^{\text {Karakas }}, \lambda_{\min }\right)$. 


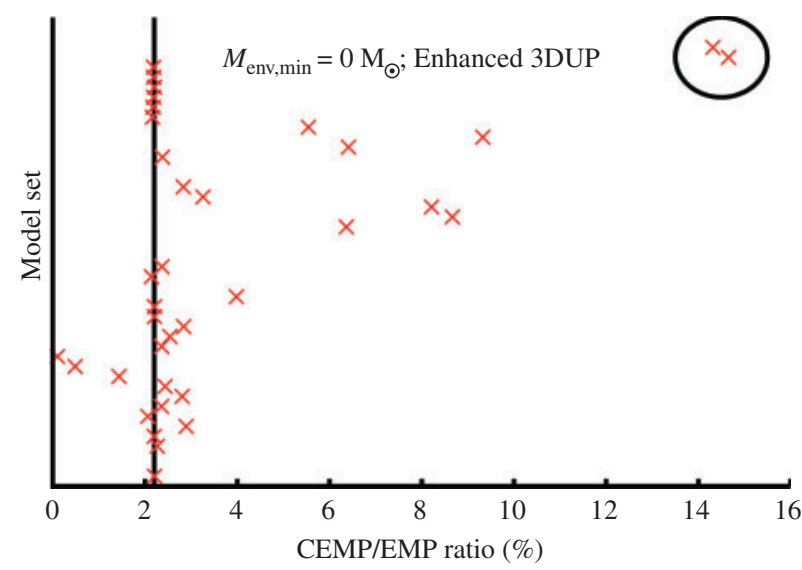

Figure 1 The CEMP/EMP ratio for our simulated binary populations. The abscissa is the CEMP/EMP ratio while the ordinate is chosen arbitrarily to separate the models with different physical parameters. The black vertical line at $2.2 \%$ shows the CEMP/EMP ratio of our default model set.

A new parameter in our model is the minimum envelope mass a star must have in order for third dredge-up to occur, $M_{\text {env, min }}$. This was set to $0.5 \mathrm{M}_{\odot}$ in previous models, following Straniero et al. (1997), but will be treated as a free parameter for our purposes. Note that while our models include canonical third dredge-up and hot-bottom burning, they do not contain prescriptions for dual-core flashes or dual-shell flashes (e.g. Campbell \& Lattanzio 2008; Cristallo et al., this volume) which are expected to occur at low metallicity $([\mathrm{Fe} / \mathrm{H}] \lesssim-3)$. Our standard choice of physical parameters is described in some detail in Izzard et al. (2006) and the metallicity is, in most of our models, $Z=10^{-4}$ (such that $[\mathrm{Fe} / \mathrm{H}]=-2.3$ ). We distribute initial primary masses according to the IMF of Kroupa, Tout \& Gilmore (1993), the binary mass ratio is distributed evenly between 0 and 1 and the separation distribution is flat in log-separation between 3 and $10^{5} \mathrm{R}_{\odot}$ (the upper limit is chosen to include all CEMPs formed by wind accretion). We assume a binary fraction of $100 \%$.

We constructed dozens of binary-star populations with a wide variety of physical parameter choices, as shown in Figure 1. These include alternative TPAGB wind prescriptions, enhanced wind-accretion efficiency, tidally-enhanced mass loss, alternative commonenvelope-evolution prescriptions, accretion of material in a common-envelope phase, lower initial metallicity and various combinations of $\Delta M_{\mathrm{c}, \min }, \lambda_{\min }$ and $M_{\mathrm{env}, \min }$. Figure 1 shows that most combinations of parameters do not lead to a CEMP/EMP ratio which is anywhere near the observed values, instead most results cluster around a ratio of $2 \%$ (similar to the $\mathrm{CH}$-to-giant ratio mentioned above). We conclude that most of the free parameters in our model are not important for CEMP formation.

This conclusion is untrue for the parameters which affect third dredge-up in low-mass stars: $\Delta M_{\mathrm{c}, \min }, \lambda_{\text {min }}$ and $M_{\mathrm{env}, \min }$. A choice of $\Delta M_{\mathrm{c}, \min } \sim-0.1 \mathrm{M}_{\odot}$ reduces the minimum core mass for third dredge-up so that it can occur in low-mass stars right down to the lower mass limit of $0.85 \mathrm{M}_{\odot}$. A positive $\lambda_{\min }$ forces third dredge-up to be efficient in low-mass stars (we choose values of 0.5 and greater). Also important is $M_{\mathrm{env}, \min }$ : the default value of $0.5 \mathrm{M}_{\odot}$ prevents third dredge-up in low-mass stars because they have an envelope mass that is too small. When we set $M_{\text {env,min }}$ to zero, together with $\Delta M_{\mathrm{c}, \min } \sim-0.1 \mathrm{M}_{\odot}$ and $\lambda_{\min } \sim 0.5$, we find CEMP/EMP ratios of up to $15 \%$. These ratios are far more compatible with the observations.

We must point out that we have artificially increased the amount of third dredge-up in low-mass TP-AGB stars without any physical justification. However, new detailed stellar evolution models by Stancliffe \& Glebbeek (2008) show third dredge-up in a $0.9-\mathrm{M}_{\odot}$ star, albeit with an efficiency of only $\lambda=0.16$. Alternatively, Cristallo et al. (this volume) show that proton ingestion at a first 'huge pulse' may occur at higher metallicities than previously thought, perhaps up to $[\mathrm{Fe} / \mathrm{H}]=-2$, in stars of mass around $0.85 \mathrm{M}_{\odot}$. This mechanism also leads to an efficient third dredge-up and carbon enrichment of the stellar envelope, as our models suggest is required. Work to simulate populations of binaries which include this mechanism is underway.

\section{Tentative Conclusions}

If TP-AGB stars with masses as low as $0.85 \mathrm{M}_{\odot}$ and metallicity around $[\mathrm{Fe} / \mathrm{H}] \approx-2.3$ undergo efficient third dredge-up they may be responsible for the formation of the majority of the CEMP stars. Canonical detailed TP-AGB models do not tend to support this conclusion, as only stars with masses above about $1.2 \mathrm{M}_{\odot}$ show third dredge-up.

New detailed models suggest that third dredge-up may occur in low-mass, low-metallicity stars, possibly due to a 'huge first pulse'. Even if we include this very efficient third dredge-up in low-mass stars in our binary population models, we cannot increase the CEMP to EMP number ratio beyond $15 \%$ even with a binary fraction of $100 \%$. Perhaps a combination of efficient third dredge-up with alternative binary distributions (the IMF, mass-ratio and period distributions) is responsible (Pols et al., this volume).

\section{Acknowledgments}

R.G.I. thanks the NWO for his fellowship in Utrecht and is the recipient of a Marie Curie-Intra European Fellowship at ULB. R.J.S. is funded by the Australian Research Council's Discovery Projects scheme under grant DP0879472. $\mathrm{He}$ is grateful to Churchill College for his Junior Research Fellowship, under which this work commenced.

\section{References}

Abia, C., Domínguez, I., Gallino, R., Busso, M., Straniero, O., de Laverny, P. \& Wallerstein, G., PASA, 20, 314

Campbell, S. W. \& Lattanzio, J. C., 2008, AIPC, 990, 315

Frebel, A. et al., 2006, ApJ, 652, 1585

Hurley, J. R., Tout, C. A. \& Pols, O. R., 2002, MNRAS, 329, 897

Izzard, R. G., Dray, L. M., Karakas, A. I., Lugaro, M. \& Tout, C. A., 2006, A\&A, 460, 565 
Izzard, R. G., Tout, C. A., Karakas, A. I. \& Pols, O. R., 2004, MNRAS, 350, 407

Karakas, A. \& Lattanzio, J. C., 2007, PASA, 24, 103

Karakas, A. I., Lattanzio, J. C. \& Pols, O. R., 2002, PASA, 19, 515

Komiya, Y., Suda, T., Minaguchi, H., Shigeyama, T., Aoki, W. \& Fujimoto, M. Y., 2007, ApJ, 658, 367

Kroupa, P., Tout, C. \& Gilmore, G., 1993, MNRAS, 262, 545

Lucatello, S., Gratton, R. G., Beers, T. C. \& Carretta, E., 2005a, ApJ, 625,833

Lucatello, S., Tsangarides, S., Beers, T. C., Carretta, E., Gratton, R. G. \& Ryan, S. G., 2005b, ApJ, 625, 825
Lucatello, S., Beers, T. C., Christlieb, N., Barklem, P. S., Rossi, S., Marsteller, B., Sivarani, T. \& Lee, Y. S., 2006, ApJ, 652, L37

Luck, R. E. \& Bond, H. E., 1991, ApJS, 77, 515

Stancliffe, R. J. \& Glebbeek, E., 2008, MNRAS, 389, 1828

Straniero, O., Chieffi, A., Limongi, M., Busso, M., Gallino, R. \& Arlandini, C., 1997, ApJ, 478, 332

Suda, T., Katsuta, Y., Yamada, S., Suwa, T., Ishizuka, C., Komiya, Y., Sorai, K., Aikawa, M. \& Fujimoto, M. Y., 2008, PASJ, 60, 1159 Tsangarides, S., Ryan, S. G. \& Beers, T. C., 2004, MmSAI, 75, 772 Wallerstein, G. \& Knapp, G. R., 1998, ARA\&A, 36, 369 